\title{
Association between single nucleotide polymorphism rs11057401 of CCDC92 gene and the risk of coronary heart disease (CHD)
}

\author{
Lingyan Xiao', Dongyang Shi', Hui Zhang ${ }^{2}$, Yanchun Zhang ${ }^{3}$, Yongfu Liu' ${ }^{1}, \mathrm{Hu} \mathrm{Lu}^{1}$ and Yishan Zheng ${ }^{1 *}$
}

\begin{abstract}
Background: Given that the CCDC92 (coiled-coil domain containing 92) was important in insulin resistance, we sought to investigate whether the CCDC92 rs825476 SNP is associated with the risk of CHD in Chinese Han population.

Methods: Rs 11057401 was genotyped for 817 patients with CHD and 724 age- and sex-matched controls using PCR-based Invader assay with the probe sets designed and synthesized by third wave.

Results: Patients were found to have a significantly higher frequency of AA than the controls (23.5\% vs. $14.7 \%$, $\mathrm{OR}=1.60, p=0.000$ ), and the frequency of allele $\mathrm{A}$ was found to be remarkably higher in the patients than the controls ( $48.1 \%$ vs. $40.3 \%, \mathrm{OR}=1.19, p=0.000$ ). Multivariate logistic analysis showed that the incidence of $\mathrm{CHD}$ was positively correlated with hyperlipidemia, T2D and rs11057401 AA/AT genotypes. The FPG, TC, and ApoA1 levels in the CHD patients were different among the AA, AT and $\Pi$ genotypes $(P<0.05)$, the A allele carriers had higher FPG, TC and lower ApoA1 levels than the A allele non-carriers $(P<0.05)$.

Conclusion: The genotypic and allelic frequencies of the rs11057401 SNP were significantly different between the patients with CHD and controls. Subjects with AA genotype or A allele were associated with an increased risk of CHD. The AA/AT genotypes were also associated with increased serum FPG, TC and decreased ApoA1 in CHD.
\end{abstract}

Keywords: CCDC92 gene, Single nucleotide polymorphism, Lipids, Coronary artery disease, Ischemic stroke

\section{Background}

Coronary artery disease (CAD) is one of the most common cardiovascular disease, with high morbidity and mortality [1]. Coronary heart disease (CHD) is the most severe clinical manifestation of $\mathrm{CAD}$ and the leading cause of death worldwide [2]. CHD is a complex disease characterized by the inheritance of multiple genetic variants in addition to environmental factors which worsen the disease state $[3,4]$. Many factors have been associated with $\mathrm{CHD}$, including plasma lipid concentrations, blood pressure, smoking, diabetes and markers of inflammation. There has long been evidence from

\footnotetext{
* Correspondence: zysicu@163.com

${ }^{1}$ Intensive care unit, the Second Hospital of Nanjing, Southeast University,

Nanjing, Jiangsu 210008, China

Full list of author information is available at the end of the article
}

observational studies that those with type 2 diabetes (T2D) have an increased risk of CHD [5]. Patients with T2D are at a twofold higher risk of mortality due to CHD as compared to individuals who do not have T2D [6]. Recent Genome-wide association studies have also identified several loci associated with both T2D and CHD risk [7]. Insulin resistance plays an important role in the pathogenesis and evolution of T2D [8]. Similarly, insulin resistance has also been found to be the single most important cause of $\mathrm{CHD}$, responsible for about $42 \%$ of myocardial infarction $[9,10]$. Coiled-coil domain containing 92 (CCDC92) is a coiled-coil domain protein that interacts with proteins at the centriole-ciliary interface, and is found to be a previously unrecognized molecule influencing adipocyte differentiation [7]. Given that the CCDC92 was important in insulin resistance, 
we sought to investigate whether CCDC92 plays role in the development of CHD. The aim of this study was to determine whether the CCDC92 rs825476 SNP is associated with the risk of CHD in Chinese Han population.

\section{Methods}

\section{Subjects}

A total of 976 patients with CHD and 724 age- and sexmatched controls were recruited. CHD can be defined as including typical ischemic symptoms, plus one or more electrocardiographic changes (ST-segment depression or elevation of $\geq 0.5 \mathrm{~mm}$, T-wave inversion of $\geq 3 \mathrm{~mm}$ in $\geq 3$ leads, or left bundle branch block), in addition to increases in cardiac markers, such as creatinine kinase-MB and troponin T. Coronary angiography was carried out in patients with CHD. The CHD subjects who had significant coronary stenosis $(\geq 50 \%)$ in at least one of the three main coronary arteries or their major branches (branch diameter $\geq 2 \mathrm{~mm}$ ) were included in the study. In addition, the angiographic severity of disease was classified according to the number of coronary vessels with significant stenosis (luminal narrowing $\geq 50 \%$ ) as one-, two-, or three-vessel disease in the three major coronary arteries. All participants were unrelated Chinese and underwent biochemical testing. Patients were excluded when they had autoimmune disease, or serious chronic diseases or a history of CHD. Finally, a total of 817 patients with CHD were included (Fig. 1). In 817 patients, 243 were Non-ST elevation myocardial infarction (NSTEMI), 122 ST elevation myocardial infarction (NSTEMI), 201 unstable angina and 251 stable angina pectoris. The study was in accordance with the principles of the Declaration of Helsinki and was approved by the clinical trials and biomedical ethics committee of the Second Hospital of Nanjing, Southeast University. Written informed consent for participation in the study and the donation of samples were obtained from all participants and their legal proxies.

\section{Biochemical analysis}

Venous blood samples were collected from all subjects after at least $12 \mathrm{~h}$ of fasting. The following information including fasting plasma glucose (FPG, $\mathrm{mmol} / \mathrm{L}$ ), triglycerides (TG, mmol/L), total cholesterol (TC, $\mathrm{mmol} / \mathrm{L}$ ), low-density lipoprotein cholesterol (LDL-C, $\mathrm{mmol} / \mathrm{L}$ ), high-density lipoprotein cholesterol (HDL-C, mmol/L), Apolipoprotein A1 (ApoA1, g/L), Apolipoprotein B (ApoB, g/L) smoking habits, and chronic diseases containing hypertension and T2D. FPG and lipid/lipoprotein levels were determined using standard laboratory techniques. The diagnosis of hypertension was made if patients were under treatment or the mean blood pressure of 3 measurements was $>140 / 90 \mathrm{mmHg}$. T2DM was diagnosed according to the World Health Organization

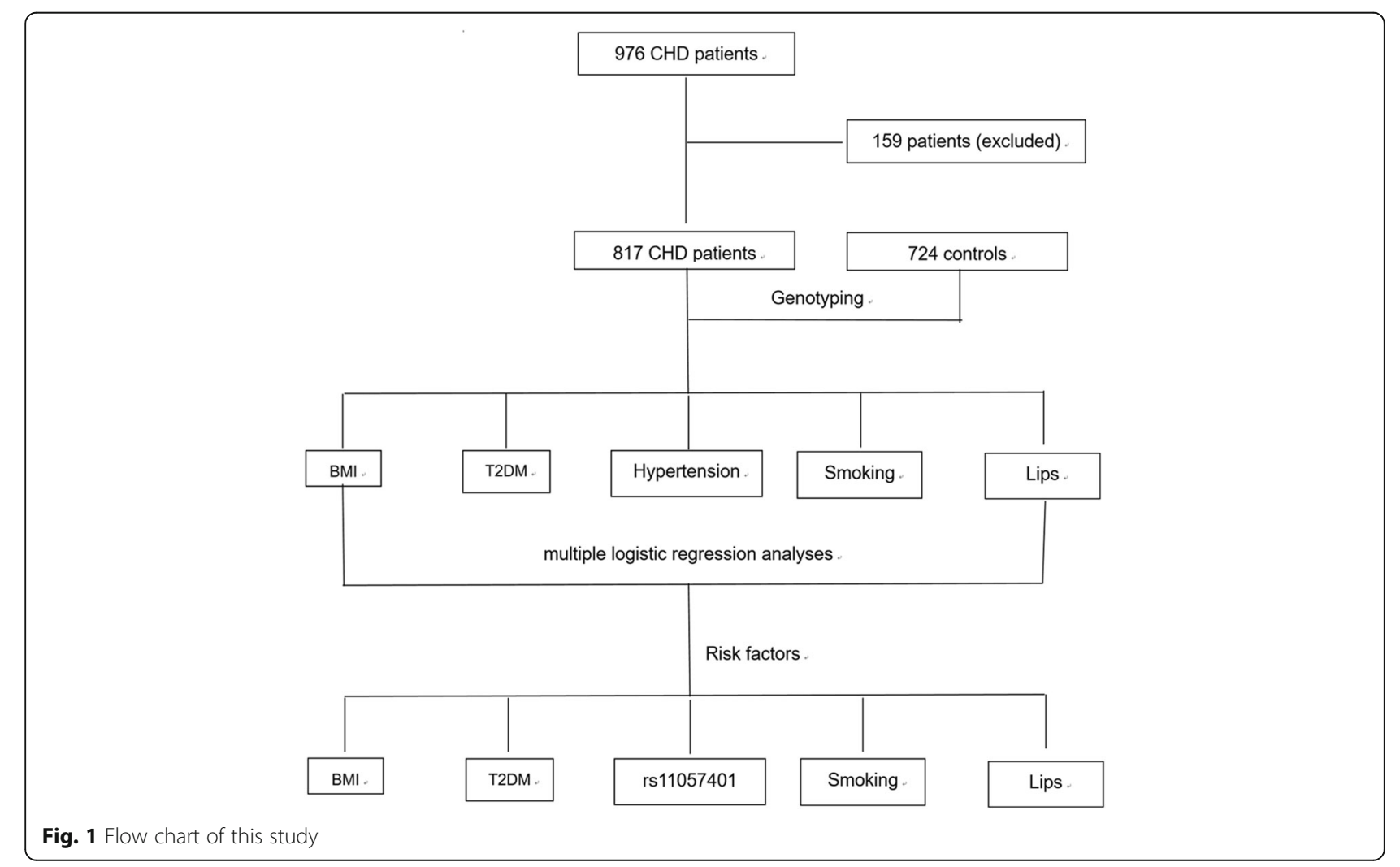


Table 1 Comparison of general characteristics between controls and CHD patients

\begin{tabular}{llll}
\hline & CHD & Control & $P$ \\
\hline Male/Female & $571 / 246$ & $523 / 201$ & 0.311 \\
Age (years) & $64.3 \pm 10.6$ & $63.7 \pm 11.2$ & 0.428 \\
BMI (kg/m²) & $23.7 \pm 3.1$ & $22.9 \pm 3.2$ & 0.572 \\
FPG (mmol/L) & $6.42 \pm 1.57$ & $6.15 \pm 1.43$ & 0.000 \\
TC (mmol/L) & $4.57 \pm 1.21$ & $4.49 \pm 0.62$ & 0.001 \\
TG (mmol/L) & $1.53 \pm 1.64$ & $1.62 \pm 1.37$ & 0.327 \\
LDL-C (mmol/L) & $2.97 \pm 0.68$ & $2.74 \pm 0.82$ & 0.000 \\
HDL-C (mmol/L) & $1.20 \pm 0.43$ & $1.89 \pm 0.39$ & 0.000 \\
ApoA1 (g/L) & $1.03 \pm 0.27$ & $1.42 \pm 0.35$ & 0.000 \\
ApoB (g/L) & $1.01 \pm 0.25$ & $0.97 \pm 0.36$ & 0.312 \\
ApoA1/ApoB & $1.01 \pm 0.44$ & $1.57 \pm 0.57$ & 0.027 \\
Smoking, n (\%) & $296(36.2 \%)$ & $237(33.6 \%)$ & 0.273 \\
T2DM, $n(\%)$ & $180(22.0 \%)$ & $123(17.0 \%)$ & 0.013 \\
Hypertension, n (\%) & $355(43.5 \%)$ & $240(33.1 \%)$ & 0.000 \\
\hline
\end{tabular}

LDL-C Low-density lipoprotein cholesterol, HDL-C High-density lipoprotein cholesterol, TC Total cholesterol, TG Triglyceride, FPG fasting plasma glucose, ApoA1 Apolipoprotein A1, ApoB Apolipoprotein B

criteria or had a documented clinical diagnosis of T2DM from medical records. The individuals with $\mathrm{TC}>$ $5.17 \mathrm{mmol} / \mathrm{L}$ and/or TG $>1.70 \mathrm{mmol} / \mathrm{L}$ were defined as hyperlipidemic.

\section{Genotyping of SNP}

Rs11057401 was genotyped using PCR-based Invader assay with the probe sets designed and synthesized by third wave. The genotyping results were read with an ABI PRISM7900HT sequence detection system (Applied Biosystems, Foster City, CA). Twenty percent of the samples were selected randomly to validate the reproducibility of the genotyping results.

\section{Statistical analysis}

Data were analyzed using SPSS software (SPSS Inc., Chicago, IL, USA, version 13.0). Chi square test was used to examine differences of allele frequencies and genotype distributions between CHD patients and controls. Unconditional multiple logistic regression analyses were employed to estimate unadjusted and adjusted odds ratios (ORs) and 95\% confidence intervals (CIs). Adjusted ORs and $P$ values were corrected for factors associated with CHD that included sex, age, body mass index (BMI) and smoking status, T2D, hypertension and hyperlipidemia. The association between genotypes and clinical parameters was tested by analysis of covariance (ANCOVA). Statistical significance was determined by a $P$ value $<0.05$. All $P$ values were two-sided.

\section{Results}

\section{General characteristics}

The levels of FPG, TC, LDL-C, and the percentages of T2D and hypertension were higher but the concentrations HDL-C, ApoA1 and the ApoA1 to ApoB ratio were lower in persons who suffered from $\mathrm{CHD}$ than in controls $(P<0.05)$. There were no significant differences of TG, ApoB, BMI, and smoking habits between the two groups $(P>0.05)$ (Table 1$)$.

\section{Genotypic and allelic frequencies}

No significant difference regarding the genotype frequency in the patients and the controls was showed by HWE test. Patients were found to have a significantly higher frequency of AA than the controls $(23.5 \%$ vs. $14.7 \%$, OR $=1.60, p=0.000$ ), and the frequency of allele A was found to be remarkably higher in the patients than the controls ( $48.1 \%$ vs. $40.3 \%, \mathrm{OR}=1.19, p=0.000)$. (Table 2).

\section{Related risk factors for $\mathrm{CHD}$}

Multivariate logistic analysis showed that the incidence of CHD was positively correlated with hyperlipidemia, T2D and rs11057401 AA/AT genotypes (Table 3).

\section{Genotypes and clinical parameters}

The FPG, TC, and ApoA1 levels in the CHD patients were different among the $\mathrm{AA}, \mathrm{AT}$ and $\mathrm{TT}$ genotypes $(P<0.05)$, the A allele carriers had higher FPG, TC and lower ApoA1 levels than the A allele non-carriers $(P<0.05)$ (Table 4$)$.

\section{Discussion}

Reduced insulin-stimulated glucose metabolism in skeletal muscles (insulin resistance) and hyperinsulinism have been found the common features in a widespread of diseases, being implicated in adverse health outcomes such as hypertension, cardiovascular disease, cerebrovascular disease, peripheral vascular disease (atherosclerosis),

Table 2 Allele and genotype frequencies of rs11057401 for CHD patients and normal controls

\begin{tabular}{|c|c|c|c|c|c|c|c|c|c|c|c|}
\hline \multirow[t]{2}{*}{ Genotype } & \multicolumn{2}{|l|}{$\mathrm{CHD}$} & \multicolumn{2}{|c|}{ Control } & \multirow[t]{2}{*}{$X 2(P)$} & \multirow[t]{2}{*}{ Allele } & \multicolumn{2}{|l|}{$\mathrm{CHD}$} & \multicolumn{2}{|c|}{ Control } & \multirow[t]{2}{*}{$x^{2}(P)$} \\
\hline & $\bar{N}$ & $\%$ & $\mathrm{~N}$ & $\%$ & & & $\bar{n}$ & $\%$ & $n$ & $\%$ & \\
\hline$\overline{A A}$ & 175 & 23.5 & 103 & 14.7 & 0.000 & $A$ & 680 & 48.1 & 566 & 40.3 & 0.000 \\
\hline AT & 330 & 44.3 & 360 & 51.2 & & $\mathrm{~T}$ & 734 & 51.9 & 840 & 59.7 & \\
\hline TT & 202 & 32.2 & 240 & 34.1 & & & & & & & \\
\hline
\end{tabular}


Table 3 The relative risk factors for CHD

\begin{tabular}{|c|c|c|}
\hline Relative factors & OR $(95 \%$ CI)) & $P$ \\
\hline Male & 1 & \\
\hline Female & $1.10(0.95-1.27)$ & 0.418 \\
\hline Age $<60$ years & 1 & \\
\hline Age $\geq 60$ years & $1.14(0.93-1.31)$ & 0.362 \\
\hline Nonsmoking & 1 & \\
\hline Smoking & $1.73(1.26-2.34)$ & 0.009 \\
\hline $\mathrm{BMI}<24 \mathrm{~kg} / \mathrm{m}^{2}$ & 1 & \\
\hline $\mathrm{BMI} \geq 24 \mathrm{~kg} / \mathrm{m}^{2}$ & $1.95(1.46-2.50)$ & 0.006 \\
\hline rs11057401 Tा & 1 & \\
\hline rs11057401 AA/AT & $1.61(1.21-2.16)$ & 0.012 \\
\hline No T2DM & 1 & \\
\hline T2DM & $1.52(1.17-1.93)$ & 0.017 \\
\hline Normotensive & 1 & \\
\hline Hypertension & $1.17(0.92-1.47)$ & 0.383 \\
\hline Normal blood lips & 1 & \\
\hline Hyperlipidemia & $2.13(1.73-2.81)$ & 0.005 \\
\hline
\end{tabular}

LDL-C Low-density lipoprotein cholesterol, HDL-C High-density lipoprotein cholesterol, TC Total cholesterol, TG Triglyceride, FPG fasting plasma glucose, ApoA1 Apolipoprotein A1, ApoB Apolipoprotein B

congestive heart failure, non-alcoholic fatty liver disease, android obesity and a variety of malignancies $[11,12]$. After correction for traditional risk factors like hypertension, hyperlipidaemia and family history, insulin resistance has been considered as an independent risk factor for ischemic heart diseases $[13,14]$ and represent a major underlying abnormality driving cardiovascular diseases [15].

The pathogenic mechanism by which insulin resistance is thought to induce CHD is said to be through its direct atherogenic action on vessel wall cells and indirectly through upper body obesity and lipids homeostasis [16]; and it has been associated with higher levels of triglycerides, lower levels of HDL-C, elevated blood pressure, non-insulin dependent diabetes mellitus (NIDDM) [17]. A recent GWAS study showed that genetically mediated increase in T2D risk also confers higher CHD risk [7]. Moreover, joint T2D-CHD analysis identified eight variants-two of which are coding-where T2D and CHD associations appear to colocalize, including a new joint T2D-CHD association at the CCDC92 locus that also replicated for T2D. Klarin et al. [8] performed a GWAS in UK Biobank testing $~ 9$ million DNA sequence variants for association with coronary artery disease and carried out meta-analysis with previously published results, identifying 15 new loci. They observed significant association for CCDC92 p.Ser70Cys (rs11057401) with body fat percentage and waist-to-hip circumference ratio, as well as plasma HDL, triglyceride, and adiponectin levels. The directionalities of these associations are hallmarks of insulin resistance and lipodystrophy, and the association with plasma adiponectin levels localizes these genetic effects to adipose tissue. Chasman et al. [18] conducted a GWAS, and demonstrated genetic influences of CCDC92 gene on concentration and size of LDL, HDL, and VLDL particles. Lotta et al. [19] conducted an integrative genomic analysis providing evidence that loci of CCDC92 gene influence adipose gene expression, leading to impaired adipogenesis, reduced peripheral fat depots and, ultimately, increased risk of cardiac disease. The aforementioned studies have strongly indicated the important role of CCDC92 in insulin resistance, which would further influence CHD risk. In addition to genetic factors, nutraceuticals play a bigger role on dyslipidemia and cardiovascular diseases. Nutraceuticals and functional food ingredients that are beneficial to vascular health may represent useful compounds that are able to reduce the overall cardiovascular risk induced by dyslipidemia by acting parallel to statins or as adjuvants in case of failure or in situations where statins cannot be used [20].

Our study demonstrated that the CCDC92 gene could influence the levels of serum lipid and glucose, which would add the risks of CHD. Patients with A allele had higher levels of FPG, TC, and lower ApoA1 as well as increased risks of $\mathrm{CHD}$ when compared to those with $\mathrm{T}$ allele. In addition to abnormal lipid metabolism, insulin resistance was also an important risk factor for CHD. Previous studies have repeatedly approved the role of

Table 4 Clinical characteristics of the patients according to the rs11057401 polymorphism in the CCDC92 gene

\begin{tabular}{lllllllll}
\hline & FPG $(\mathrm{mmol} / \mathrm{L})$ & $\mathrm{TC}(\mathrm{mmol} / \mathrm{L})$ & $\mathrm{TG}(\mathrm{mmol} / \mathrm{L})$ & $\mathrm{LDL}-\mathrm{C}(\mathrm{mmol} / \mathrm{L})$ & $\mathrm{HDL}-\mathrm{C}(\mathrm{mmol} / \mathrm{L})$ & ApoA1 $(\mathrm{g} / \mathrm{L})$ & ApoB $(\mathrm{g} / \mathrm{L})$ & ApoA1 $/ \mathrm{ApoB}$ \\
\hline TT & $6.29 \pm 1.62$ & $4.40 \pm 1.16$ & $1.51 \pm 1.72$ & $2.95 \pm 0.70$ & $1.21 \pm 0.46$ & $1.05 \pm 0.31$ & $1.02 \pm 0.28$ & $1.03 \pm 0.49$ \\
AT & $6.35 \pm 1.47$ & $4.56 \pm 1.37$ & $1.54 \pm 1.62$ & $2.98 \pm 0.65$ & $1.22 \pm 0.41$ & $1.04 \pm 0.25$ & $1.01 \pm 0.21$ & $1.02 \pm 0.42$ \\
AA & $6.47 \pm 1.51$ & $4.63 \pm 1.04$ & $1.55 \pm 1.54$ & $2.99 \pm 0.66$ & $1.19 \pm 0.45$ & $1.01 \pm 0.26$ & $0.98 \pm 0.24$ & $0.99 \pm 0.43$ \\
P & 0.021 & 0.026 & 0.371 & 0.392 & 0.427 & 0.038 & 0.621 & 0.205 \\
$\Pi \pi$ & $6.29 \pm 1.62$ & $4.40 \pm 1.16$ & $1.51 \pm 1.72$ & $2.95 \pm 0.70$ & $1.21 \pm 0.46$ & $1.05 \pm 0.31$ & $1.02 \pm 0.28$ & $1.03 \pm 0.49$ \\
AA+AT & $6.42 \pm 1.48$ & $4.61 \pm 1.27$ & $1.54 \pm 1.59$ & $2.98 \pm 0.67$ & $1.20 \pm 0.39$ & $1.02 \pm 0.24$ & $1.00 \pm 0.25$ & $1.00 \pm 0.43$ \\
P & 0.038 & 0.017 & 0.231 & 0.428 & 0.518 & 0.029 & 0.462 & 0.379 \\
\hline
\end{tabular}

LDL-C Low-density lipoprotein cholesterol, HDL-C High-density lipoprotein cholesterol, TC Total cholesterol, TG Triglyceride, FPG fasting plasma glucose, ApoA1 Apolipoprotein A1, ApoB Apolipoprotein B 
abnormal lipid metabolism in the development of CHD. Our study revealed that insulin resistance related gene also pose effects on CHD, implying shared etiological pathways of CHD with T2D.

\section{Conclusions}

This study revealed that the genotypic and allelic frequencies of the rs11057401 SNP were significantly different between the patients with $\mathrm{CHD}$ and controls. Subjects with AA genotype or A allele were associated with an increased risk of CHD. The AA/AT genotypes were also associated with increased serum FPG, TC and decreased ApoA1 in CHD.

\section{Abbreviations}

ANCOVA: Analysis of covariance; Apo: Apolipoprotein; ApoA1: Apolipoprotein A1; ApoB: Apolipoprotein B; BMl: Body mass index; CCDC92: Coiled-coil domain containing 92; CHD: Coronary heart disease; FPG: Fasting plasma glucose; GWAS: Genome-wide association study; HDL-C: High-density lipoprotein cholesterol; HWE: Hardy-Weinberg equilibrium; LDL-C: Lowdensity lipoprotein cholesterol; PCR: Polymerase chain reaction; SNP: Single nucleotide polymorphism; T2DM: Type 2 diabetes mellitus; TC: Total cholesterol; TG: Triglyceride

\section{Acknowledgements}

Not applicable

\section{Funding}

Not applicable

\section{Availability of data and materials}

The datasets generated during the present study are not publicly available, because detailed genetic information of each participant was included in these materials.

\section{Authors' contributions}

ZY conceived the study, participated in the design, undertook genotyping, performed the statistical analyses, and drafted the manuscript. XL conceived the study, participated in the design, carried out the epidemiological survey, collected the samples, and helped to draft the manuscript. SD, LH and LY collaborated to the genotyping. ZY and ZH carried out the epidemiological survey, and collected the samples. All authors read and approved the final manuscript.

\section{Ethics approval and consent to participate}

The study design was approved by the Ethics Committee of the Second Hospital of Nanjing, Southeast University. Informed consent was obtained from all participants.

\section{Consent for publication}

Informed consent including consent to publish was obtained from all participants by signature or by fingerprint (to express consent), as approved by the ethical review committee.

\section{Competing interests}

The authors declare that they have no competing interests.

\section{Publisher's Note}

Springer Nature remains neutral with regard to jurisdictional claims in published maps and institutional affiliations.

\section{Author details}

${ }^{1}$ Intensive care unit, the Second Hospital of Nanjing, Southeast University, Nanjing, Jiangsu 210008, China. ${ }^{2}$ Department of Endocrinology, the Affiliated Hospital of Xuzhou Medical University, Xuzhou, Jiangsu 221003, China. ${ }^{3}$ Department of Cardiology, the Second Hospital of Huai'an, Xuzhou Medical University, Huai'an, Jiangsu 223002, China.
Received: 11 January 2018 Accepted: 31 January 2018

Published online: 13 February 2018

\section{References}

1. Pfisterer ME, Zellweger MJ, Gersh BJ. Management of stable coronary artery disease. Lancet. 2010;375(9716):763-72.

2. Heusch G, Libby P, Gersh B, Yellon D, Bohm M, Lopaschuk G, et al. Cardiovascular remodelling in coronary artery disease and heart failure. Lancet. 2014:383(9932):1933-43.

3. Batty GD, Shipley M, Smith GD, Kivimaki M. Long term risk factors for coronary heart disease and stroke: influence of duration of follow-up over four decades of mortality surveillance. Eur J Prev Cardiol. 2015;22(9):1139-45.

4. Dhindsa DS, Khambhati J, Sandesara PB, Eapen DJ, Quyyumi AA. Biomarkers to predict cardiovascular death. Card Electrophysiol Clin. 2017;9(4):651-64.

5. Ray KK, Seshasai SR, Wijesuriya S, Sivakumaran R, Nethercott S, Preiss D, et al Effect of intensive control of glucose on cardiovascular outcomes and death in patients with diabetes mellitus: a meta-analysis of randomised controlled trials. Lancet. 2009;373(9677):1765-72.

6. Ahmad OS, Morris JA, Mujammami M, Forgetta V, Leong A, Li R, et al. A Mendelian randomization study of the effect of type-2 diabetes on coronary heart disease. Nat Commun. 2015;6:7060.

7. Zhao W, Rasheed A, Tikkanen E, Lee JJ, Butterworth AS, Howson JMM, et al. Identification of new susceptibility loci for type 2 diabetes and shared etiological pathways with coronary heart disease. Nat Genet. 2017:49(10):1450-7.

8. Klarin D, Zhu QM, Emdin CA, Chaffin M, Horner S, McMillan BJ, et al. Genetic analysis in UK biobank links insulin resistance and transendothelial migration pathways to coronary artery disease. Nat Genet. 2017;49(9):1392-7.

9. Inoue T, Matsunaga R, Sakai Y, Yaguchi I, Takayanagi K, Morooka S. Insulin resistance affects endothelium-dependent acetylcholine-induced coronary artery response. Eur Heart J. 2000;21(11):895-900.

10. Hedblad B, Nilsson P, Engstrom G, Berglund G, Janzon L. Insulin resistance in non-diabetic subjects is associated with increased incidence of myocardial infarction and death. Diabet Med. 2002;19(6):470-5.

11. Pedersen $\mathrm{O}$. Insulin resistance-a physiopathological condition with numerous sequelae: non-insulin-dependent diabetes mellitus (NIDDM), android obesity, essential hypertension, dyslipidemia and atherosclerosis. Ugeskr Laeger. 1992;154(20):1411-8.

12. Ausk KJ, Boyko EJ, loannou GN. Insulin resistance predicts mortality in nondiabetic individuals in the U.S. Diabetes Care. 2010;33(6):1179-85.

13. Burchfiel CM, Sharp DS, Curb JD, Rodriguez BL, Abbott RD, Arakaki R, et al. Hyperinsulinemia and cardiovascular disease in elderly men: the Honolulu heart program. Arterioscler Thromb Vasc Biol. 1998;18(3):450-7.

14. Pyorala M, Miettinen $\mathrm{H}$, Halonen $\mathrm{P}$, Laakso M, Pyorala K. Insulin resistance syndrome predicts the risk of coronary heart disease and stroke in healthy middle-aged men: the 22-year follow-up results of the Helsinki policemen study. Arterioscler Thromb Vasc Biol. 2000;20(2):538-44.

15. Ginsberg HN. Insulin resistance and cardiovascular disease. J Clin Invest. 2000;106(4):453-8.

16. Dongerkery SP, Schroeder PR, Shomali ME. Insulin and its cardiovascular effects: what is the current evidence? Curr Diab Rep. 2017;17(12):120.

17. Mayer EJ, Newman B, Quesenberry CP Jr, Friedman GD, Alcohol SJV. Consumption and insulin concentrations. Role of insulin in associations of alcohol intake with high-density lipoprotein cholesterol and triglycerides. Circulation. 1993:88(5 Pt 1):2190-7.

18. Chasman DI, Pare G, Mora S, Hopewell JC, Peloso G, Clarke R, et al. Fortythree loci associated with plasma lipoprotein size, concentration, and cholesterol content in genome-wide analysis. PLoS Genet. 2009;5(11): e1000730.

19. Lotta LA, Gulati P, Day FR, Payne F, Ongen $H$, van de Bunt $M$, et al. Integrative genomic analysis implicates limited peripheral adipose storage capacity in the pathogenesis of human insulin resistance. Nat Genet. 2017; 49(1):17-26

20. Scicchitano P, Cameli M, Maiello M, Modesti PA, Muiesan ML, Novo S, et al. Nutraceuticals and dyslipidaemia: beyond the common therapeutics. J Funct Foods. 2014;6(1):11-32 